\title{
On designing dielectric elastomer actuators
}

\section{Citation}

Moscardo, Mickael, Xuanhe Zhao, Zhigang Suo, and Yuri Lapusta. 2008. “On Designing Dielectric Elastomer Actuators." Journal of Applied Physics 104 (9): 93503. https:// doi.org/10.1063/1.3000440.

\section{Permanent link}

http://nrs.harvard.edu/urn-3:HUL.InstRepos:41467437

\section{Terms of Use}

This article was downloaded from Harvard University's DASH repository, and is made available under the terms and conditions applicable to Other Posted Material, as set forth at http:// nrs.harvard.edu/urn-3:HUL.InstRepos:dash.current.terms-of-use\#LAA

\section{Share Your Story}

The Harvard community has made this article openly available.

Please share how this access benefits you. Submit a story.

\section{Accessibility}




\title{
On designing dielectric elastomer actuators
}

\author{
Mickael Moscardo, ${ }^{1,2}$ Xuanhe Zhao, ${ }^{1}$ Zhigang Suo, ${ }^{1, a)}$ and Yuri Lapusta ${ }^{2}$ \\ ${ }^{1}$ School of Engineering and Applied Sciences, Harvard University, Cambridge, Massachusetts 02138, USA \\ ${ }^{2}$ IFMA-LAMI, French Institute of Advanced Mechanics, Campus de Clermont-Ferrand/Les Cezeaux, \\ 63175 Aubière, France
}

(Received 3 July 2008; accepted 26 August 2008; published online 4 November 2008)

\begin{abstract}
Subject to a voltage, a dielectric elastomer can deform substantially, making it a desirable material for actuators. Designing such an actuator, however, has been challenging due to nonlinear equations of state, as well as multiple modes of failure, parameters of design, and measures of performance. This paper explores these issues, using a spring-roll actuator as an example. We formulate the equations of state with two degrees of freedom and describe the constraints due to several modes of failure of the elastomer, including electrical breakdown, electromechanical instability, loss of tension, and tensile rupture. Also included is the compressive limit of the spring. We show that, for the spring-roll actuator, loss of tension in the axial direction will always precede electromechanical instability. We then describe a procedure to maximize the range of actuation by choosing the parameters of design, such as the prestretch of the elastomer and the stiffness of the spring. (C) 2008 American Institute of Physics. [DOI: 10.1063/1.3000440]
\end{abstract}

\section{INTRODUCTION}

Dielectric elastomer actuators have been intensely studied in the recent decade. ${ }^{1-15}$ Possible applications include medical devices, energy harvesters, and space robotics. ${ }^{16-27}$ The essential part of such an actuator is a membrane of a dielectric elastomer sandwiched between two compliant electrodes. When a voltage is applied between the electrodes, the elastomer reduces its thickness and expands its area, converting electrical energy into mechanical energy. Attractive features of dielectric elastomer actuators include large strain $(>200 \%)$, fast response $(<1 \mathrm{~ms})$, and high efficiency $(80 \%-90 \%)^{2}$

Many types of dielectric elastomer actuators have been proposed. ${ }^{18-27}$ The performance of a given type of actuators can be markedly enhanced by a judicious choice of the parameters of design. However, choosing the parameters of design to optimize performance has been challenging, due to the nonlinear equations of state, as well as multiple modes of failure, parameters of design, and measures of performance. In the literature, the choice of the parameters of design has been mostly made by experimental trial and error.

To explore some of the basic issues in design, we study one particular type of actuators, the spring-roll actuators. ${ }^{23-26}$ The construction of a spring-roll actuator is sketched in Fig. 1. Two membranes of a dielectric elastomer are alternated with two electrodes. The laminate is prestretched in two directions in the plane, and then rolled around a spring. When the actuator is subjected to an applied voltage and an applied axial force, the axial elongation couples the electrical and mechanical actions. The parameters of design include the prestretches of the elastomer and the stiffness of the spring. We will formulate equations of state and describe several

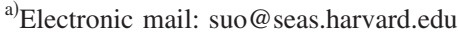

modes of failure. We then specify a measure of performance, the range of actuation, and choose the parameters of design to optimize the actuator.

\section{EQUATIONS OF STATE}

This section models the spring-roll actuator as a thermodynamic system with two degrees of freedom. We identify generalized coordinates, loading parameters, and parameters
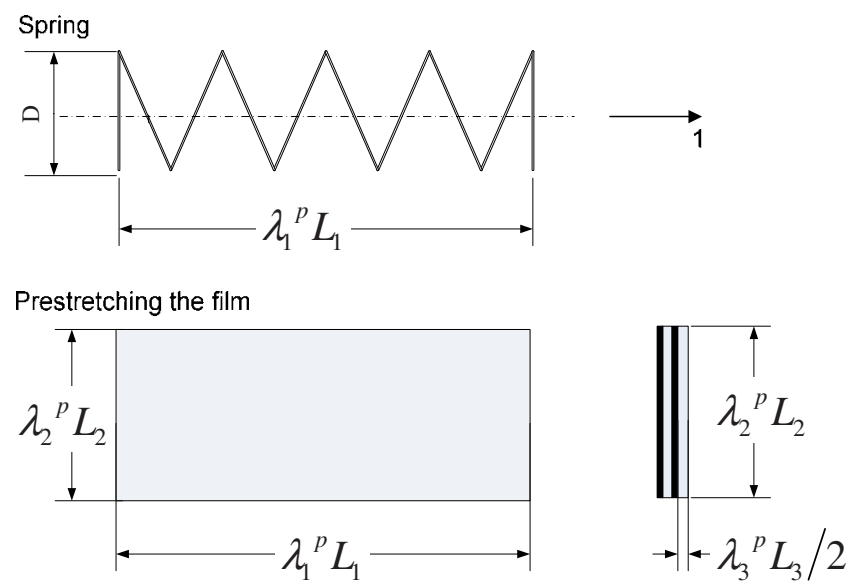

Spring Roll Actuator

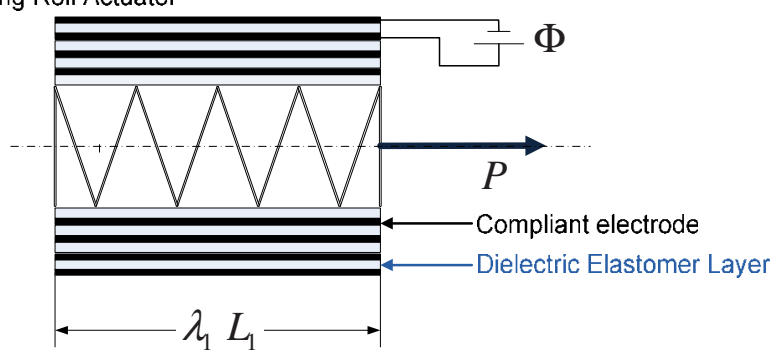

FIG. 1. (Color online) The construction of a spring-roll actuator. Two membranes of a dielectric elastomer are alternated with two electrodes. The laminate is first prestretched and then rolled around a relaxed spring. When the spring roll is subjected to a voltage and an axial force, the length of the spring couples the electrical and mechanical actions. 
of design. We then prescribe the free energy of the actuator as a function of the generalized coordinates and derive the equations of state. Following a long tradition of thermodynamics, we represent the equations of state graphically on the plane spanned by the generalized coordinates.

With reference to Fig. 1, the electrodes are compliant and bear no mechanical load. The relaxed elastomer is of thickness $L_{3}$ and sides $L_{2}$ and $L_{1}$. The relaxed spring is of length $\lambda_{1}^{p} L_{1}$. In fabrication, to fit side 1 of the elastomer to the length of the spring, one may choose to either prestretch the elastomer, or precompress the spring, or do some combination of both. Evidently, this choice in fabrication should not affect the actuator. Figure 1 illustrates the first choice, where one prestretches the elastomer to $\lambda_{2}^{p} L_{2}$ and $\lambda_{1}^{p} L_{1}$, and then rolls the elastomer around the relaxed spring. The laminate may roll around the spring several times, but the total thickness of the elastomer in the roll is taken to be small compared to the diameter of the spring, so that the state of deformation in the elastomer is homogenous from the innermost round to the outermost round. When the actuator is subject to an applied voltage $\Phi$ and an applied axial force $P$, the thickness of the laminate changes to $\lambda_{3} L_{3}$, and the length of the spring changes to $\lambda_{1} L_{1}$. However, side 2 of the laminate $\lambda_{2}^{p} L_{2}$ is constrained by the diameter of the spring and remains unchanged. The elastomer is taken to be incompressible, so that $\lambda_{1} \lambda_{2}^{p} \lambda_{3}=1$.

During operation, the actuator varies its state in two ways, as specified by two generalized coordinates: the stretch $\lambda_{1}$ in the axial direction and the charge $Q$ on one of the electrodes. We assume the actuator to be held at a constant temperature and prescribe the Helmholtz free energy $A$ of the actuator as a function of the two generalized coordinates

$$
\begin{aligned}
A\left(\lambda_{1}, Q\right)= & \frac{\mu}{2}\left(\lambda_{1}^{2}+\left(\lambda_{2}^{p}\right)^{2}+\left(\lambda_{1} \lambda_{2}^{p}\right)^{-2}-3\right) L_{1} L_{2} L_{3} \\
& +\frac{1}{2 \varepsilon}\left(\frac{Q}{\lambda_{1} L_{1} \lambda_{2}^{p} L_{2}}\right)^{2} L_{1} L_{2} L_{3}+\frac{1}{2} k\left(\lambda_{1} L_{1}-\lambda_{1}^{p} L_{1}\right)^{2} .
\end{aligned}
$$

In prescribing the free-energy function (1), we have invoked several idealizations. First, the elastomer is taken to be a cross-linked network of long and flexible polymers, obeying the Gaussian statistics, ${ }^{28}$ with $\mu$ being the shear modulus of the elastomer. Second, following Refs. 14, 15, and 20, we assume that the dielectric behavior of the elastomer is liquidlike, unaffected by the deformation, so that the free energy of the elastomer is the sum of the elastic energy and the dielectric energy, with $\varepsilon$ being the permittivity of the elastomer. Third, the spring is taken to obey Hooke's law, with $k$ being the stiffness of the spring. Of course, a spring-roll actuator in reality may deviate from these idealizations. Any such deviation can be accounted for by modifying the free-energy function, but should not alter the procedure of analysis described below.

When the actuator is in a state $\left(\lambda_{1}, Q\right)$, in equilibrium with the applied force $P$ and the applied voltage $\Phi$, for any small change in the stretch and charge $d \lambda_{1}$ and $d Q$, the change in the Helmholtz free energy equals the work done by the applied force and the voltage, namely, ${ }^{29}$

$$
d A=P L_{1} d \lambda_{1}+\Phi d Q
$$

Consequently, the force and the voltage are the partial differential coefficients of the free-energy function $A\left(\lambda_{1}, Q\right)$. The axial force is work conjugate to the elongation

$$
P=\frac{\partial A\left(\lambda_{1}, Q\right)}{L_{1} \partial \lambda_{1}} .
$$

The voltage is work conjugate to the charge

$$
\Phi=\frac{\partial A\left(\lambda_{1}, Q\right)}{\partial Q} .
$$

Inserting (1) into (3), we obtain that

$$
\begin{aligned}
\frac{P}{\mu L_{2} L_{3}}= & \left(\lambda_{1}-\lambda_{1}^{-3}\left(\lambda_{2}^{p}\right)^{-2}\right)-\frac{1}{\lambda_{1}^{3}\left(\lambda_{2}^{p}\right)^{2}}\left(\frac{Q}{\sqrt{\mu \varepsilon} L_{1} L_{2}}\right)^{2} \\
& +\alpha\left(\lambda_{1}-\lambda_{1}^{p}\right),
\end{aligned}
$$

where $\alpha=\left(k L_{1}\right) /\left(\mu L_{2} L_{3}\right)$ is a dimensionless ratio between the stiffness of the spring and that of the elastomer. Equation (5) shows that the axial force is balanced by contributions of three origins: the elasticity of the elastomer, the permittivity of the elastomer, and the elasticity of the spring. Equation (5) can also be obtained by invoking the Maxwell stress. ${ }^{23-26}$

Inserting (1) into (4), we obtain that

$$
\frac{\Phi}{L_{3}} \sqrt{\frac{\varepsilon}{\mu}}=\frac{1}{\left(\lambda_{1} \lambda_{2}^{p}\right)^{2}}\left(\frac{Q}{\sqrt{\mu \varepsilon} L_{1} L_{2}}\right) .
$$

Equation (6) recovers the familiar equation for an incompressible dieletric liquid $D=\varepsilon E$, where $E=\Phi /\left(\lambda_{3} L_{3}\right)$ is the true electric field and $D=Q /\left(\lambda_{1} L_{1} \lambda_{2}^{p} L_{2}\right)$ is the true electric displacement.

The actuator has three dimensionless parameters of design: the prestretches in the two directions in the plane of the elastomer $\lambda_{1}^{p}$ and $\lambda_{2}^{p}$, as well as the normalized stiffness of the spring $\alpha$. These parameters of design are prescribed once the actuator is constructed. Equations (5) and (6) are the equations of state, relating the dimensionless loading parameters, $P /\left(\mu L_{2} L_{3}\right)$ and $\Phi /\left(L_{3} \sqrt{\mu / \varepsilon}\right)$, to the dimensionless generalized coordinates, $\lambda_{1}$ and $Q /\left(L_{1} L_{2} \sqrt{ } \varepsilon \mu\right)$.

These nonlinear equations of state can be displayed graphically on a plane spanned by the two dimensionless generalized coordinates (Fig. 2). Plotted on this plane are the lines of constant force and the lines of constant voltage. When the applied force is fixed, both the axial stretch and the charge increase with the voltage. When the voltage is fixed, both the axial stretch and the charge increase with the applied force. Figure 2 can be used to locate the state of the actuator under prescribed axial force and voltage. For example, in the absence of the external loads, $P=0$ and $\Phi=0$, the charge also vanishes and the intersection between the line of zero force $P=0$ and the vertical axis $Q=0$ gives the stretch $\lambda_{1} \approx 1$.4. In plotting the equations of state in Fig. 2, we have set the parameters of design to a particular set of values, as indicated in Fig. 2. 


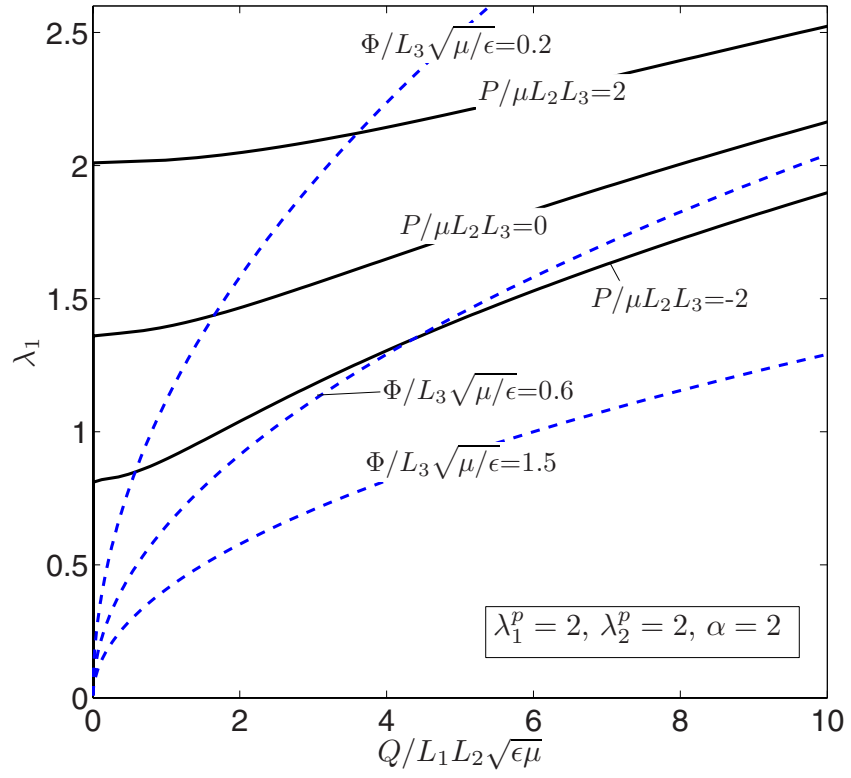

FIG. 2. (Color online) A graphic representation of the equations of state. When the design variables $\left(\alpha, \lambda_{1}^{p}, \lambda_{2}^{p}\right)$ are prescribed, the state of the actuator is characterized by two generalized coordinates: the stretch $\lambda_{1}$ in the axial direction and the charge $Q$ in one of the electrode. In the $\left(\lambda_{1}, Q\right)$ plane, a point represents a state of the actuator and a curve represents a sequence of states. Plotted on the plane are lines of constant force and lines of constant voltage.

\section{MODES OF FAILURE}

The range of operation of an actuator is limited by various modes of failure. Each mode of failure restricts the state of the actuator to a region on the plane of the generalized coordinates. The common region that averts all modes of failure constitutes the set of allowable states. To illustrate the procedure to construct the region of allowable states, we next consider several representative modes of failure. ${ }^{1,6,14}$

First we consider electromechanical instability (EMI) of the elastomer. As the applied voltage is increased, the elastomer reduces its thickness, so that the voltage induces a high electric field. The positive feedback between a thinner elastomer and a higher electric field may cause the elastomer to thin down drastically, resulting in an electrical breakdown (EB). This EMI can be analyzed by using a standard method in thermodynamics. ${ }^{14,15,30}$

Consider a three-dimensional space, with the generalized coordinates $\lambda_{1}$ and $Q$ being the horizontal axes, and the Helmholtz free energy $A$ being the vertical axis. In this space, the free-energy function $A\left(\lambda_{1}, Q\right)$ is a surface. A point on the surface represents a state of the actuator, and a curve on the surface represents a path of actuation. Imagine a plane tangent to the surface at a state $\left(\lambda_{1}, Q\right)$. The slopes of this tangent plane are $P L_{1}$ and $\Phi$, according to (3) and (4).

For a state $\left(\lambda_{1}, Q\right)$ to be stable against arbitrary small perturbation in the generalized coordinates, the surface $A\left(\lambda_{1}, Q\right)$ must be convex at point $\left(\lambda_{1}, Q\right)$. This condition of stability is equivalent to the following set of inequalities:

$$
\frac{\partial^{2} A\left(\lambda_{1}, Q\right)}{\partial \lambda_{1}^{2}}>0,
$$
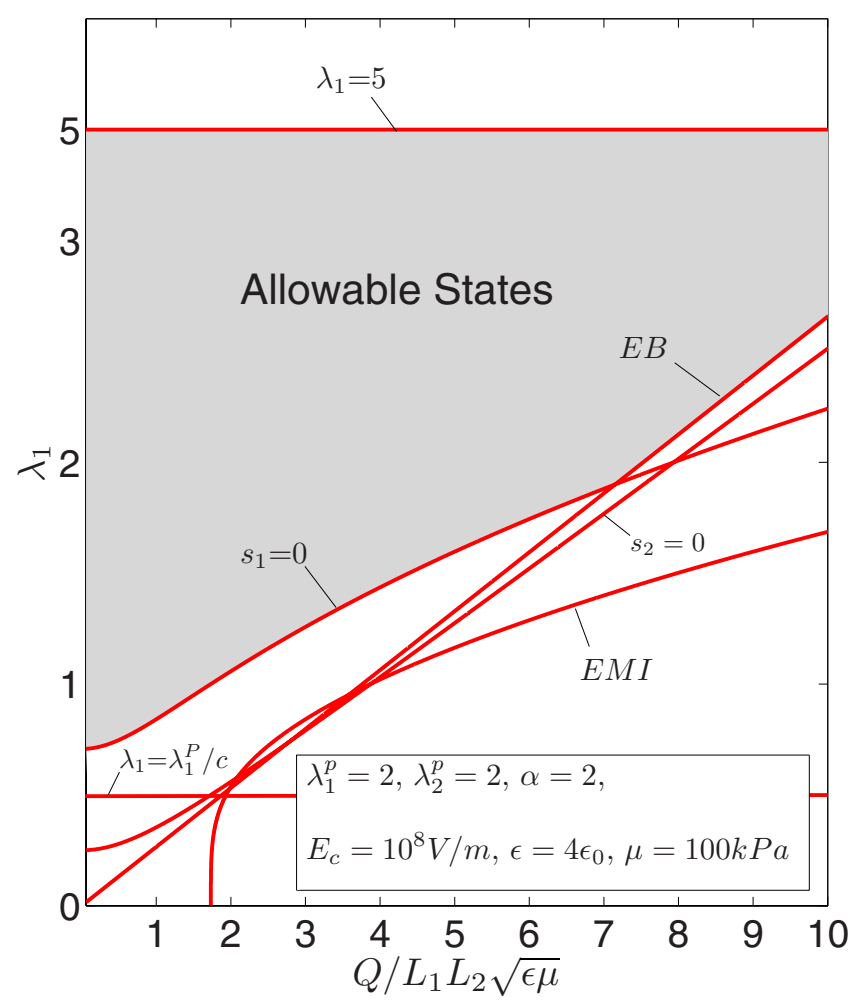

FIG. 3. (Color online) A graphic representation of modes of failure. Plotted on the $\left(\lambda_{1}, Q\right)$ plane are the critical conditions for several modes of failure: EMI, EB, loss of tension $\left(s_{1}=0\right.$ and $\left.s_{2}=0\right)$, and compressive failure of the spring $\left(\lambda_{1}=\lambda_{1}^{p} / c\right)$. All these modes of failure are averted in the shaded region marked as the allowable states. For the set of parameters of design indicated in the inset, the allowable states are bounded above by tensile rupture and bonded below by loss of tension and EB.

$$
\begin{aligned}
& \frac{\partial^{2} A\left(\lambda_{1}, Q\right)}{\partial Q^{2}}>0, \\
& \frac{\partial^{2} A\left(\lambda_{1}, Q\right)}{\partial \lambda_{1}^{2}} \frac{\partial^{2} A\left(\lambda_{1}, Q\right)}{\partial Q^{2}}>\left(\frac{\partial^{2} A\left(\lambda_{1}, Q\right)}{\partial \lambda_{1} \partial Q}\right)^{2} .
\end{aligned}
$$

Of the three inequalities, (7a) ensures mechanical stability, (7b) electrical stability, and (7c) electromechanical stability. Using (1), we can confirm that (7a) and (7b) are satisfied for all values of $\left(\lambda_{1}, Q\right)$, but (7c) is violated for some values of $\left(\lambda_{1}, Q\right)$. A combination of (1) and (7c) shows that the EMI sets in when

$$
\frac{Q}{\sqrt{\mu \varepsilon} L_{1} L_{2}}=\sqrt{(1+\alpha) \lambda_{1}^{4}\left(\lambda_{2}^{p}\right)^{2}+3} .
$$

This equation corresponds to the curve marked by EMI in Fig. 3. The curve divides the $\left(\lambda_{1}, Q\right)$ plane into two regions. Above the curve, the actuator is stable against small perturbation of the generalized coordinates. Below the curve, the actuator undergoes EMI.

We now turn to EB of the elastomer. Even before the EMI sets in, the electric field in the elastomer may become too high, leading to localized conduction path through the thickness of the elastomer. The microscopic process of EB can be complex and will not be studied in this paper. To illustrate the procedure of design, here we assume that EB occurs when the true electric field exceeds a critical value $E_{c}$. 
For the ideal dielectric elastomer $D=\varepsilon E$, where the true electric displacement is $D=Q /\left(\lambda_{1} \lambda_{2}^{P} L_{1} L_{2}\right)$, the condition for EB is

$$
\frac{Q}{\sqrt{\mu \varepsilon} L_{1} L_{2}}=\lambda_{1} \lambda_{2}^{P} E_{C} \sqrt{\frac{\varepsilon}{\mu}} .
$$

Equation (9) corresponds to the straight line marked by EB on the $\left(\lambda_{1}, Q\right)$ plane in Fig. 3. The actuator in a state in the region above this straight line will not suffer EB. The straight line in Fig. 3 is plotted by using representative values $\mu$ $=100 \mathrm{kPa}, E_{C}=10^{8} \mathrm{~V} / \mathrm{m}$, and $\varepsilon=4 \varepsilon_{0}$, with $\varepsilon_{0}=8.85$ $\times 10^{-12} \mathrm{~F} / \mathrm{m}$ being the permittivity of the vacuum.

We next consider loss of tension of the elastomer. When the voltage $\Phi$ is large or the axial force $P$ is compressive and of a large magnitude, the stress in the plane of the elastomer may cease to be tensile. This loss of tension will cause the elastomer to buckle out of the plane, so that the elastomer will no longer generate a force of actuation. To avert this mode of failure, we require that the stress be tensile in every direction in the plane of the elastomer. That is, both the stress along the axial direction and the stress in the circumferential direction are required to be tensile, $s_{1}>0$ and $s_{2}>0$. Following Ref. 14, we obtain the nominal stress in the axial direction in terms of the two generalized coordinates

$$
\frac{s_{1}}{\mu}=\left(\lambda_{1}-\lambda_{1}^{-3}\left(\lambda_{2}^{p}\right)^{-2}\right)-\left(\frac{Q}{\sqrt{\mu \varepsilon} L_{1} L_{2}}\right)^{2} \lambda_{1}^{-3}\left(\lambda_{2}^{p}\right)^{-2} .
$$

Setting the critical condition $s_{1}=0$ in (10a), we obtain that

$$
\frac{Q}{\sqrt{\mu \varepsilon} L_{1} L_{2}}=\sqrt{\lambda_{1}^{4}\left(\lambda_{2}^{p}\right)^{2}-1} .
$$

Similarly, we can obtain the nominal stress $s_{2}$ in terms of the two generalized coordinates

$$
\frac{s_{2}}{\mu}=\left(\lambda_{2}^{p}-\left(\lambda_{2}^{p}\right)^{-3} \lambda_{1}^{-2}\right)-\left(\frac{Q}{\sqrt{\mu \varepsilon} L_{1} L_{2}}\right)^{2}\left(\lambda_{2}^{p}\right)^{-3} \lambda_{1}^{-2} .
$$

Setting the critical condition $s_{2}=0$ in (11a), we obtain that

$$
\frac{Q}{\sqrt{\mu \varepsilon} L_{1} L_{2}}=\sqrt{\lambda_{1}^{2}\left(\lambda_{2}^{p}\right)^{4}-1} .
$$

The critical conditions for loss of tension, $s_{1}=0$ and $s_{2}=0$, are plotted in Fig. 3. A comparison of (8) and (10b) shows that, for spring-roll actuators, loss of tension in the axial direction will always precede EMI. By contrast, other types of dielectric elastomer actuators may fail by EMI. ${ }^{1,6,14,30}$

We next consider tensile rupture of the elastomer. When an elastomer is stretched too severely, the elastomer may rupture. The critical condition for tensile rupture is not well quantified. Here we will use the simple criterion that the elastomer will rupture when either stretch, $\lambda_{1}$ or $\lambda_{2}$, exceeds a critical value $\lambda_{c}$. A representative value $\lambda_{c}=5$ is included in Fig. 3.

We finally consider the compressive limit of the spring. The spring in the spring-roll actuator is designed to be under compression. When the spring is compressed excessively, however, it may deform plastically. The length of the spring at its relaxed state is $\lambda_{1}^{P} L_{1}$, and the length of the actuated
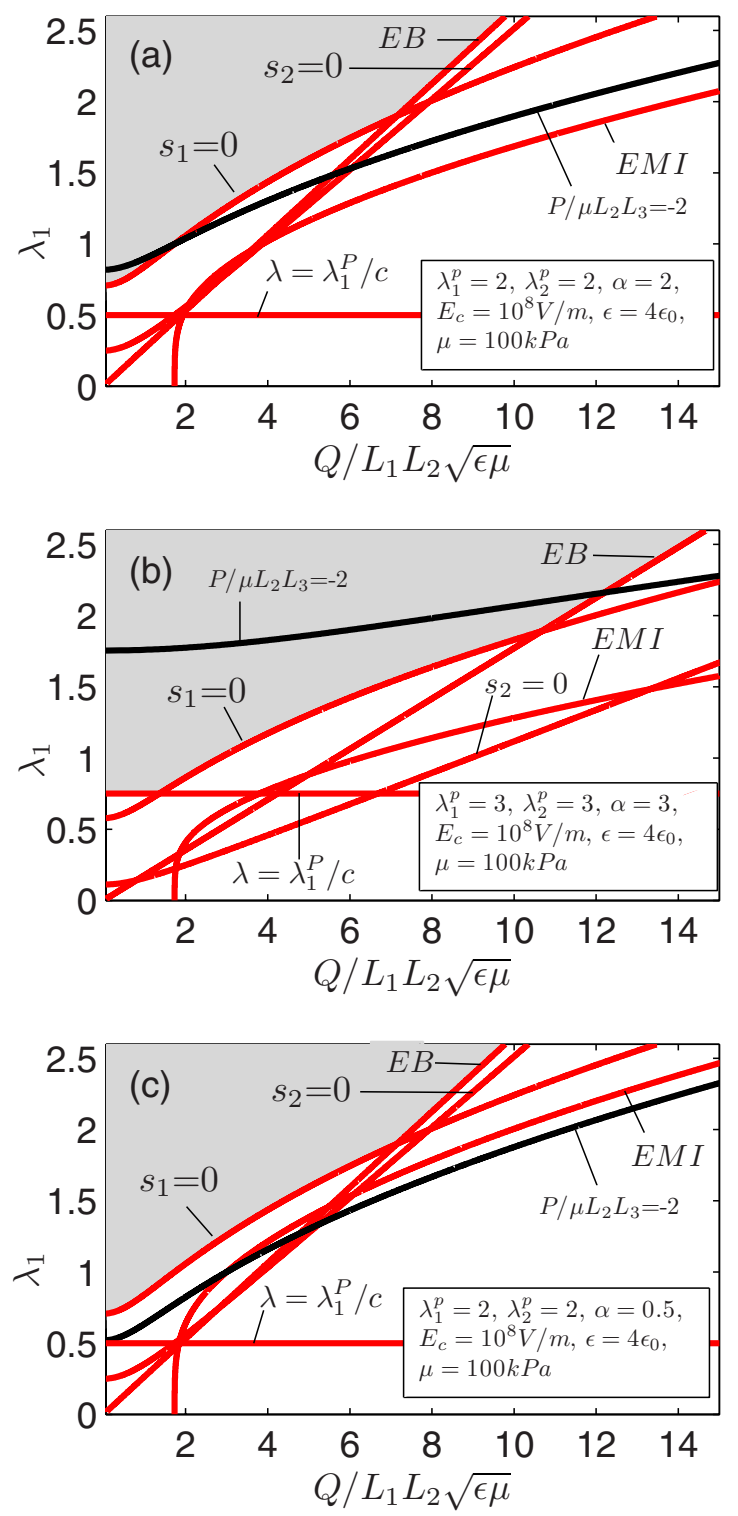

FIG. 4. (Color online) A line of constant force $P /\left(\mu L_{2} L_{3}\right)=-2$ is plotted along with the critical conditions for failure. When the voltage increases, the state of actuator moves along the line of constant force, beginning at $Q=0$ and ending at a critical state set by one mode of failure. The region of allowable states varies with the parameters of design. (a) For one set of parameters of design, the range of actuation is limited by the loss of tension in the axial direction. (b) For a second set of parameters of design, the range of actuation is limited by EB. (c) For a third set of parameters of design, the line of constant force falls outside the region of allowable states, so that the actuator so constructed will not function.

spring is $\lambda_{1} L_{1}$. We assume that the spring deforms plastically when $\lambda_{1}^{P} / \lambda_{1}$ exceeds a critical value $c$, which we set to be $c=4$. In the $\left(\lambda_{1}, Q\right)$ plane (Fig. 3) the region above the line $\lambda_{1}=\lambda_{1}^{P} / c$ will guarantee that the spring remains elastic.

The modes of failure discussed in this section are all averted in the shaded region in Fig. 3. As evident from the above discussion, this region of allowable states will depend on the critical conditions for various modes of failure. Furthermore, when a different mode of failure is identified, another curve will be added to the diagram, so that the region of allowable states may recede. In the remainder of the paper, to illustrate the procedure of design, we will confine our attention to the modes of failure and the critical conditions listed in this section. 

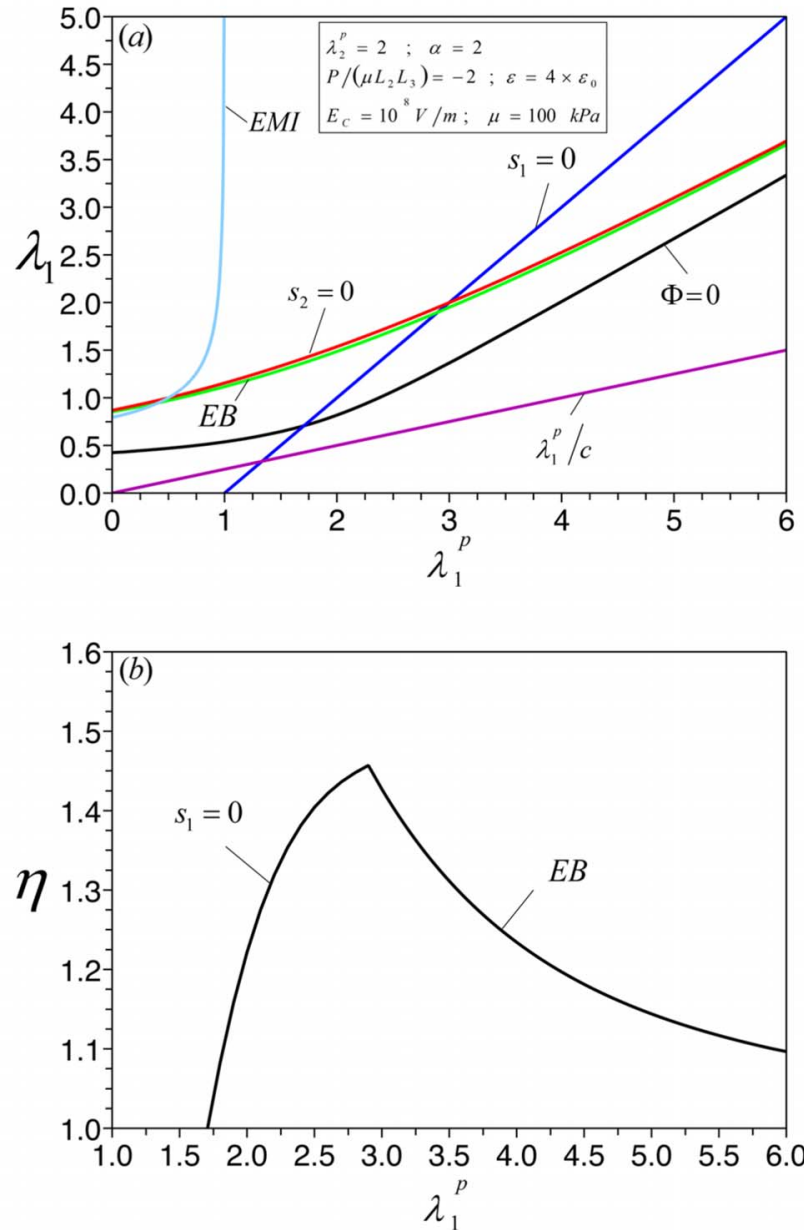

FIG. 5. (Color online) The effect of prestretch $\lambda_{1}^{p}$ on the range of actuation. (a) The values of $\lambda_{1}$ under the critical conditions for various modes of failure: EMI, EB, loss of tension $\left(s_{1}=0\right.$ and $\left.s_{2}=0\right)$, and compressive limit of the spring $\left(\lambda_{1}=\lambda_{1}^{p} / c\right)$. Also included is $\lambda_{1}$ under the condition $\Phi=0$. (b) The range of actuation as a function of $\lambda_{1}^{p}$.

\section{CHOOSE PARAMETERS OF DESIGN TO MAXIMIZE THE RANGE OF ACTUATION}

We now combine the graph for the equations of states (Fig. 2) and the graph for the modes of failure (Fig. 3). Consider an actuator subject to a fixed axial force (i.e., a dead weight), $P /\left(\mu L_{2} L_{3}\right)=-2$. Figure 4 (a) includes the line of constant force, as well as the critical conditions for various modes of failure. As the voltage increases, the state of the actuator moves along the line of constant force, starting at the state of zero charge and ending at the state when $s_{1}$ $=0$. That is, of all modes of failure considered above, loss of tension in the axial direction limits the range of operation. Diagram such as Fig. 4(a) can be constructed for other sets of parameters of design. For example, with a second set of parameters of design in Fig. 4(b), the range of operation is limited by EB. With a third set of parameters of design in Fig. 4(c), the line of constant force falls outside of the region of allowable state, indicating that the actuator so designed will not function.

How does one choose the parameters of design to optimize an actuator? To optimize an actuator, one needs to specify what is to be optimized for. That is, one needs to
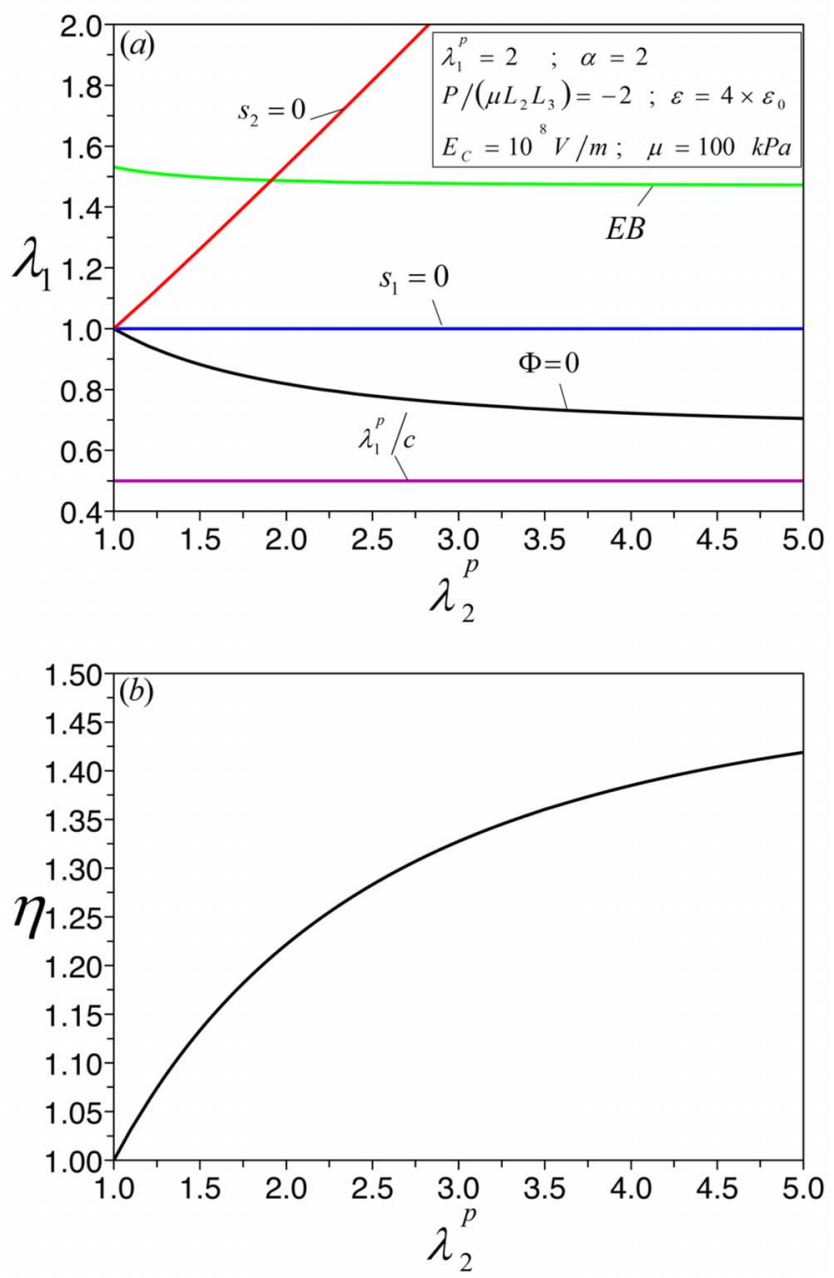

FIG. 6. (Color online) The effect of prestretch $\lambda_{2}^{p}$ on the range of actuation. (a) The values of $\lambda_{1}$ under the critical conditions for various modes of failure: $\mathrm{EB}$, loss of tension $\left(s_{1}=0\right.$ and $\left.s_{2}=0\right)$, and compressive limit of the spring $\left(\lambda_{1}=\lambda_{1}^{p} / c\right)$. Also included is $\lambda_{1}$ under the condition $\Phi=0$. For this set of parameters of design, EMI does not occur. (b) The range of actuation as a function $\lambda_{2}^{p}$.

specify a measure of performance. In practice, one might need to consider several measures of performance and choose the parameters of design to compromise. To illustrate the basic procedure, here we specify a particular measure of performance as follows.

As illustrated in Fig. 4, for a given set of the parameters of design $\left(\lambda_{1}^{p}, \lambda_{2}^{p}, \alpha\right)$ and a prescribed value of the axial load $P /\left(\mu L_{2} L_{3}\right)$ when the applied voltage increases, the state of the actuator moves along the line of constant force, starting at the state of zero charge and ending at the critical state where one failure mode sets in. We define the range of actuation $\eta$ by the stretch of the critical state with respect to the state of zero voltage, namely,

$$
\eta=\frac{\lambda_{1}^{\text {fail }}}{\left.\lambda_{1}\right|_{\Phi=0}},
$$

where $\left.\lambda_{1}\right|_{\Phi=0}$ is the axial stretch at zero voltage, and $\lambda_{1}^{\text {fail }}$ is the stretch at which the actuator fails.

The stretch at zero voltage is determined by (5) by setting $Q=0$, so that 

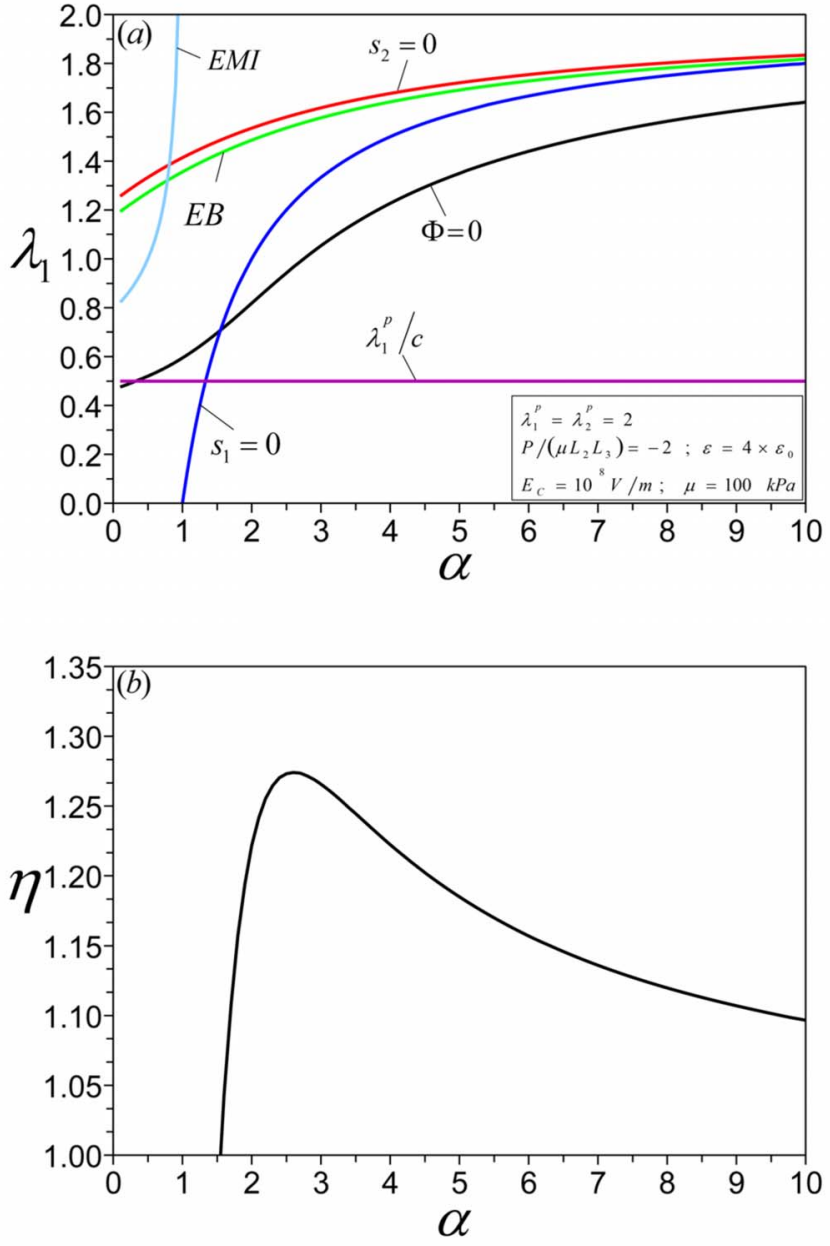

FIG. 7. (Color online) The effect of the stiffness of the spring $\alpha$ on the range of actuation. (a) The values of $\lambda_{1}$ under the critical conditions for various modes of failure: EMI, EB, loss of tension $\left(s_{1}=0\right.$ and $\left.s_{2}=0\right)$, and compressive limit of the spring $\left(\lambda_{1}=\lambda_{1}^{p} / c\right)$. Also included is $\lambda_{1}$ under the condition $\Phi=0$. (b) The range of actuation as a function of $\alpha$.

$$
\frac{P}{\mu L_{2} L_{3}}=\lambda_{1}-\lambda_{1}^{-3}\left(\lambda_{2}^{p}\right)^{-2}+\alpha\left(\lambda_{1}-\lambda_{1}^{p}\right)
$$

This nonlinear equation can be solved numerically to determine $\left.\lambda_{1}\right|_{\Phi=0}$. As illustrated by the counter example in Fig. $4(\mathrm{c})$, to ensure that the line of constant force falls inside the region of allowable states, we require that $\left.\lambda_{1}\right|_{\Phi=0}$ be above all failure criteria specified in Sec. III when $Q=0$.

The stretch $\lambda_{1}^{\text {fail }}$ is limited by one of the modes of failure, namely,

$$
\lambda_{1}^{\text {fail }}=\operatorname{Min}\left(\left.\lambda_{1}\right|_{E M I},\left.\lambda_{1}\right|_{E B},\left.\lambda_{1}\right|_{s_{1}=0},\left.\lambda_{1}\right|_{s_{2}=0}, \lambda_{c}\right) .
$$

These critical stretches are determined by inserting the various failure criteria in Sec. III into (5). The results are as follows. The critical stretch for EMI is

$$
\left.\lambda_{1}\right|_{E M I}=\left(\frac{-4 \lambda_{2}^{p^{-2}}}{\alpha \lambda_{1}^{p}+\frac{P}{\mu L_{2} L_{3}}}\right)^{1 / 3} .
$$

The critical value of $\lambda_{1}$ for EB is solved from a fourth-order algebraic equation
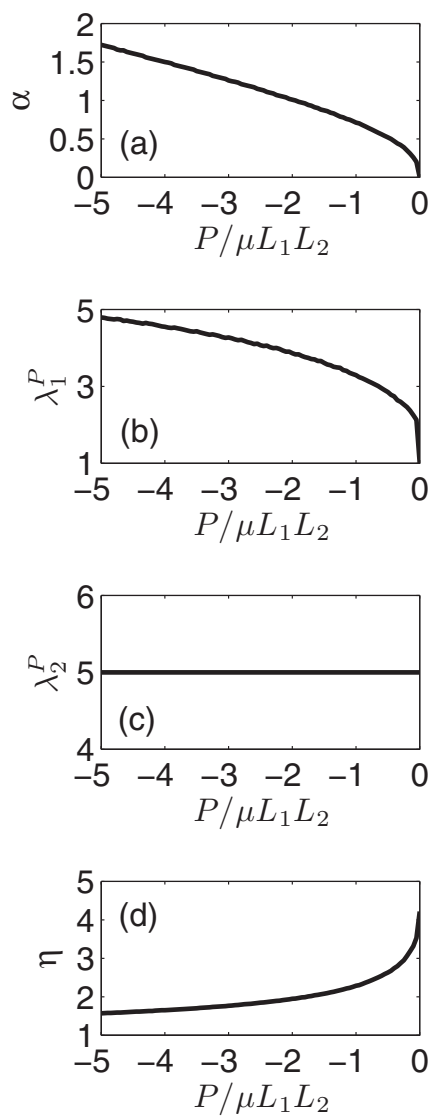

FIG. 8. The optimal design of the actuator subject to various levels of the axial force. [(a)-(c)] The optimal choice of the parameters of design. (d) The maximum range of actuation.

$$
-(1+\alpha) \lambda_{1}^{4}+\left(\alpha \lambda_{1}^{p}+\frac{P}{\mu L_{2} L_{3}}\right) \lambda_{1}^{3}+\frac{\varepsilon}{\mu} E_{C}^{2} \lambda_{1}^{2}+\left(\lambda_{2}^{p}\right)^{-2}=0 .
$$

The critical stretch for loss of tension is given by

$$
\left.\lambda_{1}\right|_{s_{1}=0}=\frac{P}{\alpha \mu L_{2} L_{3}}+\lambda_{1}^{p}
$$

and

$\left.\lambda_{1}\right|_{s_{2}=0}=\frac{\frac{P}{\mu L_{2} L_{3}}+\alpha \lambda_{1}^{p}+\sqrt{\left(\frac{P}{\mu L_{2} L_{3}}+\alpha \lambda_{1}^{p}\right)^{2}+4(\alpha+1) \lambda_{2}^{p^{2}}}}{2(\alpha+1)}$.

Now we study the effect of parameters of design on the range of actuation $\eta$. To gain insight into the behavior, we will now fix two of the three parameters of design $\left(\lambda_{1}^{p}, \lambda_{2}^{p}, \alpha\right)$ and vary the third. In addition, the axial force is held at $P /\left(\mu L_{2} L_{3}\right)=-2$. Figure 5 shows the effect of $\lambda_{1}^{p}$ on the range of actuation, plotting in Fig. 5(a) the critical stretches set by various mode of failure and in Fig. 5(b) the range of actuation. The allowable region of actuation is the region below the critical stretches and above $\left.\lambda_{1}\right|_{\Phi=0}$. As shown in Fig. 5(a), $\left.\lambda_{1}\right|_{s_{1}=0}<\left.\lambda_{1}\right|_{\Phi=0}$ when $\lambda_{1}^{p}<1.75$. For such a low prestretch, the actuator fails by loss of tension under the axial force even without any voltage. When $1.75<\lambda_{1}^{p}<3$, the loss of tension 
is still the upper limit for the range of actuation, but now the actuator can work under voltage. When $\lambda_{1}^{p}>3$, the range of actuation is limited by EB.

Figure 6 shows the effect of $\lambda_{2}^{p}$ on the range of actuation. In this case, loss of tension sets the upper limit for the range of actuation. Furthermore, the range of actuation increases monotonically with $\lambda_{2}^{p}$.

Figure 7 shows the effect of the normalized stiffness of the spring $\alpha$ on the range of actuation. In this case the loss of tension in the axial direction limits the range of actuation. The range of actuation, however, is not a monotonic function of $\alpha$. For small $\alpha$, the spring is so compliant that the elastomer loses tension readily under the dead load, and the range of actuation is small. For large $\alpha$, the spring is so stiff that the voltage cannot increase elongation much, and the range of actuation is also small. The range of actuation $\eta$ peaks at an intermediate value of $\alpha$.

Finally, we vary all three parameters of design $\left(\lambda_{1}^{p}, \lambda_{2}^{p}, \alpha\right)$ to maximize the range of actuation $\eta$, subject to the constraint of all the modes of failure. We proceed as follows. For a given value of $P /\left(\mu L_{2} L_{3}\right)$, we calculate the function $\eta\left(\lambda_{1}^{p}, \lambda_{2}^{p}, \alpha\right)$ according to the above procedure. We then locate the maximum value of $\eta$ and the associated values of $\left(\lambda_{1}^{p}, \lambda_{2}^{p}, \alpha\right)$. Inspecting the critical conditions for EMI, $\mathrm{EB}$, and loss of tension, we observe that for each of these modes the maximum allowable charge increases with $\lambda_{2}^{p}$. Consequently, in our optimization we set $\lambda_{2}^{p}$ to the maximum allowable value $\lambda_{2}^{p}=5$. Figure 8 plots the results for various level of prescribed axial force. In the absence of the applied force $P /\left(\mu L_{2} L_{3}\right)=0$, the combination of $\lambda_{1}^{p}=1, \lambda_{2}^{p}=5$, and $\alpha=0$ gives the optimal range of actuation $\eta \approx 4$.2. As the compressive force increases, one needs to increase both $\lambda_{1}^{p}$ and $\alpha$ to maximize the range of actuation.

\section{CONCLUDING REMARKS}

This paper describes an approach to design dielectric elastomer actuators to optimize performance. Using the spring-roll actuator as an illustration, we construct the equations of state by modeling the actuator as a thermodynamic system of two degrees of freedom, identify a set of design variables, and determine the region of allowable states according to several modes of failure. We then specify a measure of performance, the range of actuation, and choose the design parameters to optimize the actuator. This approach may be adapted in practice to other types of dielectric elastomer actuators, different modes of failure, other parameters of design, and other measures of performance.

\section{ACKNOWLEDGMENTS}

This research was supported by the Army Research Office through Contract No. W911NF-04-1-0170 and by the National Science Foundation through the MRSEC at Harvard University. X.H.Z. acknowledges the support of the Founder's Prize of the American Academy of Mechanics, sponsored by the Robert M. and Mary Haythornthwaite Foundation.

${ }^{1}$ R. E. Pelrine, R. D. Kornbluh, and J. P. Joseph, Sens. Actuators, A 64, 77 (1998).

${ }^{2}$ R. Pelrine, R. Kornbluh, Q. B. Pei, and J. Joseph, Science 287, 836 (2000).

${ }^{3}$ Q. M. Zhang, H. Li, M. Poh, F. Xia, Z.-Y. Cheng, H. Xu, and C. Huang, Nature (London) 419, 284 (2002).

${ }^{4}$ G. Kofod, P. Sommer-Larsen, R. Kornbluh, and R. Pelrine, J. Intell. Mater. Syst. Struct. 14, 787 (2003).

${ }^{5}$ F. Carpi and D. De Rossi, IEEE Trans. Dielectr. Electr. Insul. 12, 835 (2005)

${ }^{6}$ J. S. Plante and S. Dubowsky, Int. J. Solids Struct. 43, 7727 (2006).

${ }^{7}$ E. M. Mockensturm and N. Goulbourne, Int. J. Non-Linear Mech. 41, 388 (2006).

${ }^{8}$ S. M. Ha, W. Yuan, Q. B. Pei, R. Pelrine, and S. Stanford, Adv. Mater. (Weinheim, Ger.) 18, 887 (2006).

${ }^{9}$ M. Wissler and E. Mazza, Sens. Actuators, A 134, 494 (2007).

${ }^{10}$ L. Patrick, K. Gabor, and M. Silvain, Sens. Actuators, A 135, 748 (2007).

${ }^{11}$ N. C. Goulbourne, E. M. Mockensturm, and M. I. Frecker, Int. J. Solids Struct. 44, 2609 (2007).

${ }^{12}$ G. Gallone, F. Carpi, D. De Rossi, G. Levita, and A. Marchetti, Mater. Sci. Eng., C 27, 110 (2007).

${ }^{13}$ J. S. Plante and S. Dubowsky, Smart Mater. Struct. 16, S227 (2007).

${ }^{14}$ X. H. Zhao and Z. G. Suo, Appl. Phys. Lett. 91, 061921 (2007).

${ }^{15}$ X. H. Zhao, W. Hong, and Z. G. Suo, Phys. Rev. B 76, 134113 (2007).

${ }^{16}$ Y. Bar-Cohen, J. Spacecr. Rockets 39, 822 (2002).

${ }^{17}$ X. Q. Zhang, C. Lowe, M. Wissler, B. Jahne, and G. Kovacs, Adv. Eng. Mater. 7, 361 (2005)

${ }^{18}$ Y. Sugiyama and S. Hirai, Int. J. Robot. Res. 25, 603 (2006).

${ }^{19}$ A. Wingert, M. D. Lichter, and S. Dubowsky, IEEE/ASME Trans. Mechatron. 11, 448 (2006).

${ }^{20}$ G. Kofod, M. Paajanen, and S. Bauer, Appl. Phys. A: Mater. Sci. Process. 85, 141 (2006)

${ }^{21}$ G. Kofod, W. Wirges, M. Paajanen, and S. Bauer, Appl. Phys. Lett. 90, 081916 (2007)

${ }^{22}$ K. Meijer, M. Rosenthal, and R. J. Full, Proc. SPIE 4329, 7 (2001).

${ }^{23}$ Q. Pei, R. Pelrine, S. Stanford, R. Kornbluh, and M. Rosenthal, Synth. Met. 135-136, 129 (2003).

${ }^{24}$ Q. Pei, R. Pelrine, S. Stanford, R. Kornbluh, M. Rosenthal, K. Meijer, and R. Full, Proc. SPIE 4698, 246 (2002).

${ }^{25}$ R. Zhang, P. Lochmatter, A. Kunz, and G. Kovacs, Proc. SPIE 6168, 61681T (2006)

${ }^{26}$ G. Kovacs, P. Lochmatter, and M. Wissler, Smart Mater. Struct. 16, S306 (2007).

${ }^{27}$ J. D. Madden, Science 318, 59 (2007).

${ }^{28}$ L. R. G. Treloar, The Physics of Rubber Elasticity (Clarendon, Oxford, 1975).

${ }^{29}$ Z. G. Suo, X. H. Zhao, and W. H. Greene, J. Mech. Phys. Solids 56, 467 (2008).

${ }^{30}$ A. N. Norris, Appl. Phys. Lett. 92, 026101 (2008). 